\title{
Correlation of In Vivo and In Vitro Measures of Carbonic Anhydrase IX Antigen Expression in Renal Masses Using Antibody ${ }^{124}$ I-cG250
}

\author{
Daniel A. Pryma ${ }^{1}$, Joseph A. O’Donoghue ${ }^{2}$, John L. Humm², Achim A. Jungbluth², Lloyd J. Old², Steven M. Larson², \\ and Chaitanya R. Divgi ${ }^{1}$ \\ ${ }^{1}$ University of Pennsylvania, Philadelphia, Pennsylvania; and ${ }^{2}$ Memorial Sloan-Kettering Cancer Center, New York, New York
}

\begin{abstract}
The goal of this study was to determine whether there is a potential correlation between quantification of radiolabeled macromolecular uptake in tumors determined in vivo using PET/CT and in vitro using autoradiography and $\gamma$-counting of tumor tissue. Methods: Twenty-six patients with renal masses scheduled for surgical resection received ${ }^{124}$-labeled antibody cG250. Tumor specimens obtained from resection were studied. Fifteen of these patients had clear cell cancer demonstrated by positive findings on PET/CT images and histopathology. Radioactivity in tumors was measured on PET/CT images and expressed as percentage injected dose per gram. These values were then normalized to measurements of known serum radioactivity from a venous blood sample obtained at the time of PET/CT. Comparable measurements were obtained in vitro using $\gamma$-well counting and digital autoradiography of tumor tissue. Results: There was a significant correlation between tumor radioactivity estimated in vivo and in vitro (Spearman correlation coefficient comparing normalized PET measurements with well counting of $0.84, P<0.000001$, and with autoradiography of $0.88, P<0.000001)$. PET/CT measurements of tumor uptake were lower than measurements obtained with either of the in vitro methods, and digital autoradiography resulted in the highest measurements. Conclusion: PET/CT can be reliably used to quantify radiolabeled macromolecular uptake in vivo, suggesting important implications for quantitative pharmacokinetic estimates of macromolecular biodistribution.
\end{abstract}

Key Words: ${ }^{124}$ I-cG250; immunoPET; renal cancer; autoradiography

J Nucl Med 2011; 52:535-540

DOI: 10.2967/jnumed.110.083295

$\mathbf{P}$ harmacokinetics and biodistribution in humans have traditionally been based on values derived from serum and excreta (urine or stool). Valuable information, such as assessment of metabolites, is obtained through these analyses. However, these methods do not provide any direct insight

\footnotetext{
Received Sep. 14, 2010; revision accepted Dec. 20, 2010.

For correspondence or reprints contact: Daniel A. Pryma, Division of Nuclear Medicine and Clinical Molecular Imaging, University of Pennsylvania, 3400 Spruce St., Donner 110-C, Philadelphia, PA 19104.

E-mail: daniel.pryma@uphs.upenn.edu

COPYRIGHT @ 2011 by the Society of Nuclear Medicine, Inc.
}

into tissue and organ distribution of the agent, nor do they provide information on whether the agent reaches its target in vivo.

Evidence of organ distribution has traditionally been extrapolated from rodent biodistribution studies. An understanding of kinetics at the tissue level in humans is needed in the identification of an optimum biologic dose of a targeted agent. In cancer therapy, in particular, the increasing availability of targeted biologic therapeutics demands the development of a methodology that can provide reliable, quantitative information on tumor targeting and residence time of the agent under development, because the traditional endpoint of dose-limiting toxicity is not informative with many of these agents ( 1 ). Indeed, in many phase I studies of targeted anticancer agents, early dose levels may be active and have a better therapeutic ratio over the traditionally chosen phase II dose (2). Because many targeted biologic therapeutics have markedly different distribution in other species, the ability to perform biodistribution studies in human subjects is especially needed.

Molecular imaging offers the ability to examine biodistribution and kinetics of macromolecules through serial imaging. PET/CT, in particular, has the capability of accurately quantifying the distribution of a radiolabeled agent within an organ or any tissue of interest defined on the coregistered CT scan. Such measurements, made serially, have the potential to provide information about the entire pharmacokinetics of the radiolabeled study agent, including its uptake and retention in tumors, organs, and tissues of interest $(3,4)$. The development of organ- or tumor-specific compartmental models that in turn would permit optimization of agent delivery would provide the potential for personalizing doses, thus avoiding unnecessary toxicity in patients who require a lower dose for efficacy while withholding futile therapy from those unlikely to benefit from even maximal therapy (4). Direct comparison of measurements made in vivo by PET with ex vivo measurements of radioactivity from surgical samples retrieved after the scan has been minimal. Such verification and validation are essential for the establishment of noninvasive quantitative molecular imaging as a tool for optimizing drug delivery development. 
Our group has developed and consequently studied antibody G250, which targets the tumor-associated protein carbonic anhydrase IX (CAIX), since the late 1980s (5-9). Using single-photon imaging, we gathered valuable information for clear cell renal cancer targeting. However, quantification using single-photon imaging relied on tissue sampling by biopsy for information on specificity and extent of antibody targeting to the tumor. The development of ${ }^{124}$ I-labeled chimeric antibody cG250 enabled PET/CT and permitted us to explore, in a systematic manner, the ability of PET/CT to quantify radiolabeled antibody in tissue.

A pilot phase I study to evaluate the utility of ${ }^{124} \mathrm{I}-\mathrm{cG} 250$ $\mathrm{PET} / \mathrm{CT}$ in the detection of clear cell renal cancer was undertaken at Memorial Sloan-Kettering Cancer Center after submission of an Investigational New Drug Application to the Food and Drug Administration (BB-IND-8851) and institutional review board approval. The clinical results of that study have been published (7). We analyzed the tumoral distribution of cG250 in these patients by comparative evaluation of measured radioactivity in the tumor using PET/CT, $\gamma$-well counting, and digital autoradiography. The results of the quantitative analyses are presented here.

\section{MATERIALS AND METHODS}

\section{Patient Population}

An open-label, single-center pilot study of preoperative PET/CT with radiolabeled chimeric antibody ${ }^{124} \mathrm{I}-\mathrm{cG} 250$ in patients with indeterminate renal masses was performed. Patients scheduled for resection of a renal mass were eligible. Exclusion criteria were life expectancy less than 3 mo, Karnofsky performance status score less than 70\%, unacceptable baseline hematologic and chemical parameters, ongoing toxicity from prior therapy, pregnancy, concurrent illness precluding or limiting the amount of antibody administered, New York Heart Association class III or IV cardiac disease, autoimmune hepatitis, and iodine allergy.

\section{Antibody Preparation and Administration}

Antibody cG250 was radiolabeled on the day of infusion as previously described (7). The dose was diluted in $50 \mathrm{~mL}$ of $5 \%$ human serum albumin and administered intravenously over 20 $\mathrm{min}$. Patients were monitored during and at $2 \mathrm{~h}$ after the infusion, then periodically for $4 \mathrm{wk}$ after surgery. Toxicity was assessed according to the National Cancer Institute Common Terminology Criteria for Adverse Events (version 3.0; available at: http://ctep. cancer.gov/protocoldevelopment/electronic_applications/docs/ ctcaev3.pdf).

\section{PET/CT}

Less than $2 \mathrm{~h}$ after completion of ${ }^{124} \mathrm{I}-\mathrm{cG} 250$ infusion, PET of the abdomen was performed using an Advance (GE Healthcare) scanner in 2-dimensional mode (axial field of view [FOV], $15.2 \mathrm{~cm}$; transaxial resolution, $4.2 \mathrm{~mm}$ in full width at half maximum at the center). Acquisition was for 6 min per bed position. A rod source transmission scan was obtained for attenuation correction for $3 \mathrm{~min}$ per bed position. The PET FOV ranged from the hepatic dome to the iliac crests, ensuring that the entire known renal mass was within the FOV. Images were reconstructed with an ordered-subset expectation maximization iterative reconstruction algorithm with segmented attenuation correction.
Within $3 \mathrm{~h}$ before surgery (and $5 \pm 2 \mathrm{~d}$ after infusion), a PET/CT scan of the abdomen was acquired. All day-of-surgery images were obtained on a Discovery LS (GE Healthcare) scanner, except for 1 patient whose images were acquired using the Advance scanner. The Discovery LS consists of the Advance scanner coupled to a 4-slice Lightspeed (GE Healthcare) CT scanner. Scanner calibration was confirmed and adjusted according to clinical use standards; the calibration accuracy of ${ }^{124} \mathrm{I}$ was confirmed for a variety of activity levels and source distributions. The CT scans were obtained with tube voltage of $140 \mathrm{kVp}$, tube current of $40-85 \mathrm{~mA}$ (depending on patient weight), and maximum spatial resolution of $0.32 \mathrm{~mm}$. CT images were acquired without oral or intravenous contrast and were reconstructed with filtered backprojection into 4.3-mm axial slices to match the PET emission image slice thickness. PET images were acquired over the same FOV as the early ( $2 \mathrm{~h}$ after injection) scan, for a duration of 10 min per axial FOV. The longer scan time was partially to compensate for the loss of counts from radioactive decay and biologic clearance of the agent. Reconstruction parameters were selected to match the GE Advance values. Attenuation correction was performed using the CT attenuation maps.

PET/CT images were reviewed, and the known renal mass was identified on the CT portion of the study. Binding of ${ }^{124} \mathrm{I}-\mathrm{cG} 250$ to the renal mass was compared with neighboring normal renal parenchyma, both qualitatively (7) and quantitatively in terms of activity per unit volume $(\mathrm{Bq} / \mathrm{mL})$. Volumes of interest were drawn on the $\mathrm{CT}$ scan and transferred to their corresponding location on the PET component of the PET/CT examination. The maximum uptake, mean uptake, and SD within the region of interest were recorded in $\mathrm{Bq} / \mathrm{mL}$. The percentage injected dose per gram of tissue $(\% \mathrm{ID} / \mathrm{g})$ was calculated using 100 times the mean activity in the renal mass in $\mathrm{Bq} / \mathrm{mL}$ divided by the decay-corrected injected dose.

Immediately before the presurgical PET/CT scan, a venous blood sample was withdrawn and the serum concentration of antibody was measured as $\% \mathrm{ID} / \mathrm{L}$ and converted to $\% \mathrm{ID} / \mathrm{g}$. Then, a region of interest was drawn within the aorta on the PET/CT images, and radioactivity in the blood pool was measured in $\mathrm{Bq} / \mathrm{mL}$. The 2 measurements were used to determine a recovery coefficient relating measurements of activity within blood compartments identified on the images to the actual activity per unit blood volume measured in a well scintillation counter.

\section{Surgical Resection}

Surgical resection was performed according to the standard of care. The entire tumor mass was resected en bloc and immediately transferred to the Pathology Department. Tumor and grossly healthy-appearing renal tissue were removed for the purposes of this study, after ensuring that sufficient and appropriate tissue had been obtained for normal clinical interpretation. The tumor and normal-tissue samples were weighed and counted in a well counter to determine $\% \mathrm{ID} / \mathrm{g}$ within the specimens. Then, the tumor and normal-tissue specimens were bisected, and 1 part was embedded in optimal-cutting-temperature compound (Tissue Tek; Fischer Scientific) and snap-frozen. The other specimen half was fixed in $10 \%$ neutral-buffered formalin. After radioisotope decay, formalin-fixed tissues were then embedded in paraffin. For morphologic analysis, 5 - $\mu \mathrm{m}$ sections of frozen and paraffin blocks were placed on slides (Superfrost Plus; Menzel). Serial sections were used to compare quantitative autoradiography, immunohistochemistry, and conventional tissue morphology. For morphologic analysis, hematoxylin and eosin staining was performed on frozen and corresponding paraffin sections. 


\section{Quantitative Autoradiography}

Three 8- $\mu \mathrm{m}$ sections from each specimen were placed in a cassette against a Fujifilm BAS-MS2325 imaging plate (Fuji Photo Film Co.). Plates were exposed to the samples for a variable time ( $\sim 24 \mathrm{~h}$ ) before signal readout on a Fujifilm BAS-1800II Bio-Imaging Analyzer (Fuji Photo Film Co.) at a scanning laser resolution of $50 \mu \mathrm{m}^{2}$ per pixel. To convert counts to specific activity $(\mathrm{Bq} / \mathrm{g})$, samples were coexposed to each phosphor plate alongside 2 standards: ${ }^{14} \mathrm{C}$ and ${ }^{124} \mathrm{I}$. The ${ }^{14} \mathrm{C}$ autoradiographic standards were used to normalize between different sample exposure durations and potential differences in sensitivity of the individual plates used in the study. ${ }^{124}$ I standards were made in the laboratory by dissolving a known activity of ${ }^{124}$ I into a known weight of molten optimalcutting-temperature compound, which, after mixing, was frozen into a block and sectioned alongside the clinical specimens in the same $8-\mu \mathrm{m}$ section thickness. These sections allowed the machine readout parameter of photostimulable luminescence (PSL) per square millimeter to be converted to units of $\mathrm{Bq} / \mathrm{g}$, by direct scaling of the samples to the standard readout signal times the conversion factor $\left(\mathrm{PSL} / \mathrm{mm}^{2}\right.$ per $\mathrm{Bq} / \mathrm{g}$ ). From the specific activity within the autoradiographic specimen, the \% ID/g could be calculated. Heterogeneity of the activity within the malignant specimen was measured as SD of the mean activity.

\section{Immunohistochemistry}

Immunohistochemical detection of the CAIX antigen was performed as previously described (10). Briefly, frozen tissue sections were incubated with a murine anti-CAIX antibody (G250). Primary antibodies were detected with a biotinylated horse-antimouse secondary followed by an avidin-biotin system (Vector-Elite; Vector Labs). Scoring of antigen expression was based on the amount of immunopositive tumor cells and graded as follows: no staining, negative; focal, less than $5 \%$ cells positive; $1+$, more than $5 \%-25 \%$ of tumor cells positive; $2+$, more than $>25 \%-50 \%$ of tumor cells positive; $3+$, more than $50 \%-75 \%$ of tumor cells positive; or $4+$, more than $75 \%$ of tumor cells positive.

\section{Statistical Analysis}

PET/CT-measured \%ID/g was correlated with well-counted $\% \mathrm{ID} / \mathrm{g}$ and autoradiography-measured $\% \mathrm{ID} / \mathrm{g}$. The correlation was described with the Spearman correlation coefficient.

\section{RESULTS}

The binary accuracy of ${ }^{124} \mathrm{I}-\mathrm{cG} 250$ PET/CT in predicting clear cell renal cancer has been previously reported (7). Sen- sitivity for detection of clear cell renal cancer was $94 \%$, and specificity was $100 \%$. Day-of-infusion scans were obtained for all but 1 patient (who refused) and showed only the expected blood-pool distribution of antibody (data not shown). All patients underwent day-of-surgery imaging. However, 1 patient received antibody with severely compromised immunoreactivity and was excluded from analysis.

PET/CT images were negative in 10 patients, 9 of whom were found surgically to not have clear cell renal cancer (2 angiomyolipoma, 2 papillary, 2 oncocytoma, and 1 each myxoma, fibroma, and chromophobe renal cell carcinoma). Specimens retrieved from patients with negative ${ }^{124} \mathrm{I}-\mathrm{cG} 250$ PET/CT findings resulted in low sample counts by well counting and low-to-background levels of counts by digital autoradiography and did not express CAIX on immunohistochemistry.

The remaining 15 patients had positive ${ }^{124} \mathrm{I}-\mathrm{cG} 250 \mathrm{PET} /$ CT results; many of these patients revealed markedly heterogeneous distribution of antibody, whereas in others uptake was quite homogeneous (Fig. 1). Heterogeneity cannot be determined with well counting. On autoradiography, all positive samples revealed significant heterogeneity of antibody distribution, which matched well with distribution of CAIX expression as displayed by immunohistochemistry (Fig. 2).

All but 2 patients had higher measured \%ID/g by autoradiography than by well counting (Table 1). Despite this finding, there was a strong correlation between the measured values, with a Spearman coefficient of $0.95(P<0.000001)$.

The values for $\%$ ID/g mean measured on PET were lower than $\%$ ID/g measured with well counting or autoradiography. Despite the differences in absolute values, there was a correlation between PET-measured \% ID/g and that measured on well counting (Spearman coefficient, $0.77 ; P=0.000008$ ) and autoradiography (Spearman coefficient, $0.81 ; P=$ $0.000001)$. When PET measurements were normalized by the known serum concentration of radioactivity, measured values were much closer to the values measured by other methods (Table 1). Furthermore, correlation was stronger to well counting (Spearman coefficient, $0.84 ; P<0.000001$ ) and autoradiography (Spearman coefficient, 0.88; $P<0.000001$ ).

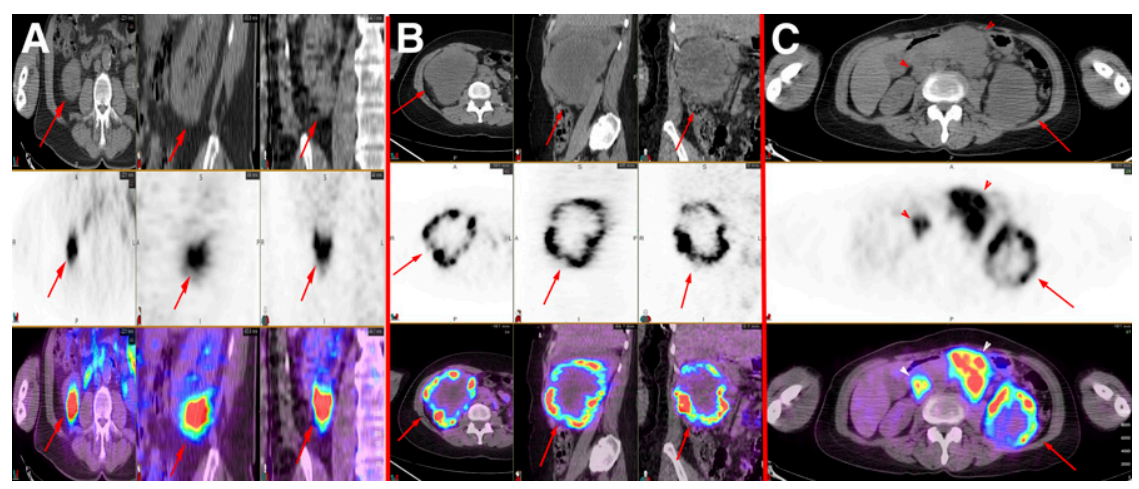

FIGURE 1. (A and B) Axial, sagittal, and coronal CT (top), PET (middle), and fused PET/CT (bottom) images of patient with clear cell renal cancer with relatively homogeneous distribution of antigen (arrows) (A) and patient with large, centrally necrotic clear cell renal cancer with marked heterogeneity of antigen distribution within mass (arrows) (B). (C) Axial CT (top), PET (middle), and fused PET/CT (bottom) images of patient with advanced clear cell renal cancer. Antigen distribution within primary tumor is heterogeneous (arrows), whereas distribution within metastatic nodes is relatively homogeneous (arrowheads). 


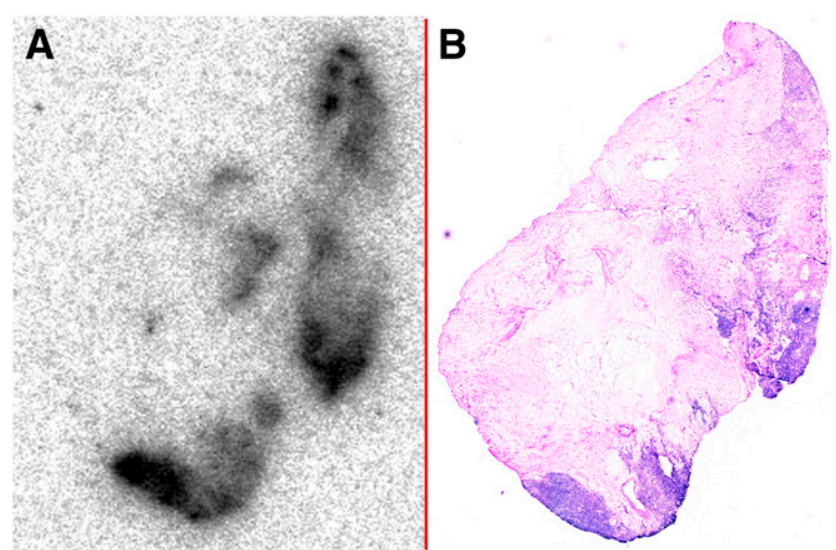

FIGURE 2. Autoradiographic (A) and histologic (B) sections from patient with clear cell renal cancer. Marked heterogeneity of antibody binding seen on autoradiography correlates with areas of densest tumor cells on histology.

Interestingly, among the most highly positive lesions, the PET measurements, compared with the other methods, were the lowest. These were the lesions with the highest SD of the means on both autoradiography and PET, indicating the greatest degree of heterogeneity of radioactivity within the tumor.

\section{DISCUSSION}

This is the first, to our knowledge, systematic, prospective study comparing ${ }^{124}$ I radioactivity measurement by in vivo PET/CT with in vitro $\gamma$-counting and digital autoradiography in 26 patients with renal masses undergoing resection. Quantification of CAIX antigen expression in vivo using ${ }^{124} \mathrm{I}-$ cG250 PET/CT agreed well with both in vitro measurements, with strongly significant correlation among the various measurements.

Patients with the highest uptake of ${ }^{124} \mathrm{I}-\mathrm{cG} 250$ as determined by PET showed the highest estimates of tumor radioactivity by well scintillation counting and digital autoradiography and, in particular, were among those exhibiting the greatest CAIX antigen expression by immunohistochemistry. The agreement between radiolabeled antibody and antigen spatial distribution and intensity increased at the microscopic level, confirming that the antibody selectively localized to the target CAIX expression sites. Activity per unit volume measurements performed by PET are obtained from 4- $\mathrm{mm}^{3}$ voxels, representing approximately $0.06 \mathrm{~g}$ of tissue. The specimens obtained from surgery used for well scintillation counting ranged from 0.05 to $0.8 \mathrm{~g}$. From these specimens, frozen tissue sections of only $8-\mu \mathrm{m}$ thickness were cut for digital autoradiography. Consequently, the measurements obtained with each of the techniques are the result of the differences in sampling size and resolution between the techniques.

Although the total amount of antigen present (or total amount of antibody binding) in a malignant deposit is relevant, this amount may not correlate perfectly with response
TABLE 1

Measured \%ID/g for Each Subject Using Digital Autoradiography, Well Counting, and PET/CT

\begin{tabular}{|c|c|c|c|}
\hline Subject & Autoradiography & Well counting & $\mathrm{PET} / \mathrm{CT}$ \\
\hline 1 & 0.0681 & 0.0334 & 0.0453 \\
\hline 2 & 0.0023 & 0.0015 & 0.0039 \\
\hline 3 & 0.0018 & 0.0006 & 0.0066 \\
\hline $4^{*}$ & - & - & - \\
\hline 5 & 0.0130 & 0.0094 & 0.0179 \\
\hline 6 & 0.0452 & 0.0380 & 0.0255 \\
\hline 7 & 0.1477 & 0.0657 & 0.0481 \\
\hline 8 & 0.1269 & 0.0602 & 0.0391 \\
\hline 9 & 0.0172 & 0.0413 & 0.0444 \\
\hline 10 & 0.0093 & 0.0053 & 0.0100 \\
\hline 11 & 0.1370 & 0.0662 & 0.0503 \\
\hline 12 & 0.0954 & 0.0369 & 0.0268 \\
\hline 13 & 0.0395 & 0.0124 & 0.0378 \\
\hline 14 & 0.0032 & 0.0003 & 0.0173 \\
\hline 15 & 0.0096 & 0.0039 & 0.0382 \\
\hline 16 & 0.0035 & 0.0022 & 0.0061 \\
\hline 17 & 0.0145 & 0.0043 & 0.0288 \\
\hline 18 & 0.0062 & 0.0037 & 0.0177 \\
\hline 19 & 0.0050 & 0.0021 & 0.0125 \\
\hline 20 & 0.0013 & 0.0006 & 0.0028 \\
\hline 21 & 0.0015 & 0.0012 & 0.0097 \\
\hline 22 & 0.0046 & 0.0020 & 0.0074 \\
\hline 23 & 0.0339 & 0.0258 & 0.0414 \\
\hline 24 & 0.0025 & 0.0015 & 0.0035 \\
\hline 25 & 0.0018 & 0.0008 & 0.0035 \\
\hline 26 & 0.0091 & 0.0195 & 0.0219 \\
\hline
\end{tabular}

*Subject 4 received immunologically inactive antibody and was excluded from analysis.

to a targeted therapy. A therapeutic with localized effects (e.g., a radionuclide with a short pathlength, such as an $\alpha$-particle or Auger electron emitter) will be more efficacious in a lesion in which there is uniform distribution of the therapeutic than in a lesion with regions that are without therapeutic penetration or binding if the therapeutic-absent regions are larger than the effective range of the therapeutic. Techniques such as well counting and flow cytometry do not evaluate the spatial distribution of antigen. Digital autoradiography is able to depict distribution at an exquisite level but is limited by a need for tissue specimens. The spatial resolution of modern PET/CT tomographs is sufficient to fully demonstrate targeting heterogeneity for higher-energy $\beta$-emitters such as ${ }^{90} \mathrm{Y}$, though the limits of the spatial resolution are challenged by shorter-pathlength radionuclides. However, the resolution is certainly sufficient to accurately assess dose distribution within normal organs for dosimetric purposes. The potential even exists to assess dose deposited to normal tissues at the boundary of metastatic deposits to help predict toxicity.

Through selective sampling, viable tumor tissue was used for autoradiography and well counting. Hence, there is little to no extraneous mass (that not containing viable tumor tissue) in the counted specimen used for digital autoradiog- 
raphy. These samples contain tumor cells within the host tissue stroma (as can be seen from the example section shown in Fig. 2). On the other hand, PET/CT assays the entire mass: tumor cells, as well as necrotic tissue, stroma, and vasculature that do not contain the targeted radioactivity. In fact, the most rapidly growing (and highest antigen expressing) masses have been shown to have the largest areas of necrosis, likely contributing to the disparity between PET/CT and autoradiographic determinations of activity per unit mass. Furthermore, it is possible to specifically select viable antigen-expressing tumor regions from the autoradiographic analysis, in contrast to quantitative data derived from PET image analysis, for which we know that only a fraction of the PET voxel volume will comprise tumor cells. Although PET/CT suffers from this type of partial-volume error, it has the advantage of providing a map of radioactivity distribution within the tumor and throughout the entire body and thus provides a global noninvasive analysis of molecular targeting. The sampling bias inherent in most pathologic techniques does not exist with PET/CT. Moreover, although pathologic sampling is temporally and spatially limited, PET/CT permits serial, quantitative whole-body sampling.

This study has several important implications. The ability to quantify antibody uptake in tumors and normal tissue permits a more accurate assessment of antibody biodistribution in vivo, in a noninvasive manner. Of particular importance is the ability to characterize tumor according to the presence, absence, or heterogeneity of expression of the targeted antigen. This ability to characterize the tumor is in contrast to the current invasive techniques based on the analysis of site-directed biopsy samples to identify and characterize the neoplasm on the assumption of congruent antigen expression over time and throughout disease sites. This assumption has been shown to be invalid in many patients, for example, women with breast cancer receiving hormone therapy based on receptor positivity at the time of initial resection but showing resistance to therapy (11). Most notably, changes in phenotype that confer susceptibility or resistance to a given therapy can be heterogeneous, even within lesions in a single patient (12).

Antibody therapy is continuing to be explored in various malignancies, both hematologic and nonhematologic solid cancers. Although dose determination for toxic agents is usually determined by the maximum tolerated amounts of agent, the lack of toxicity with most antibody therapies has resulted in empiric determinations (usually based on trough serum levels) of therapeutic amount of antibody. Quantification of antibody uptake in tumors and normal tissue using PET/CT should enable assessment of antibody targeting to the tumor, which, if supplemented by serial imaging, can provide full pharmacokinetic characterization of the radiotracer, for example, tumor and normal-tissue residence times. The ability to determine the impact of antibody amount on residence times and saturability of antigen sites in the tumor and the effect on normal-organ kinetics will have important implications for the estimation of the optimum biologic dose and provide vital information on scheduling and assessing the viability of repeated therapies.

Quantification in vivo also has potential in the early assessment of response to therapy. Downregulation or occupation of antigen sites after specific molecular therapy can be determined accurately and repeatedly using PET/CT. A demonstration of such change or the lack thereof after therapy will have implications for the continuation of therapy.

The relatively small sample size in this study is a limitation, as is the measurement of only tumor tissue. We, however, chose to focus on antigen-expressing tumor samples to validate the concept of quantitative antibody targeting through the correlation between in vivo PET/CT measurements and direct ex vivo radioactivity measurements and through autoradiographic spatial correlation from frozen unwashed sections with CAIX antigen immunohistochemistry. ${ }^{124}$ I, like many positron-emitting isotopes, is a complex radionuclide, and quantification will need to be validated for each mode of PET/CT acquisition and reconstruction. Comparable studies will also need to be undertaken for validation of other radionuclides.

\section{CONCLUSION}

In vivo quantification of CAIX antigen expression is possible using ${ }^{124} \mathrm{I}$ cG250 PET/CT. Furthermore, in vivo and in vitro measurements of antibody binding are well correlated. ImmunoPET may be useful in quantitatively assessing antigen targeting by antibody-based therapies, allowing for noninvasive interrogation of all sites of disease over time.

\section{ACKNOWLEDGMENTS}

We thank Neeta Pandit-Taskar and Paul Russo for their clinical contributions and Shutian Ruan for assistance with microscopic examinations. The study was supported in part by the Ludwig Institute for Cancer Research.

\section{REFERENCES}

1. Le Tourneau C, Lee JJ, Siu LL. Dose escalation methods in phase I cancer clinical trials. J Natl Cancer Inst. 2009;101:708-720.

2. Postel-Vinay S, Arkenau HT, Olmos D, et al. Clinical benefit in phase-I trials of novel molecularly targeted agents: does dose matter? Br J Cancer. 2009;100: 1373-1378.

3. Eschmann SM, Reischl G, Bilger K, et al. Evaluation of dosimetry of radioiodine therapy in benign and malignant thyroid disorders by means of iodine-124 and PET. Eur J Nucl Med Mol Imaging. 2002;29:760-767.

4. Larson SM, Pentlow KS, Volkow ND, et al. PET scanning of iodine-124-3F9 as an approach to tumor dosimetry during treatment planning for radioimmunotherapy in a child with neuroblastoma. J Nucl Med. 1992;33:20202023.

5. Divgi CR, Bander NH, Scott AM, et al. Phase I/II radioimmunotherapy trial with iodine-131-labeled monoclonal antibody G250 in metastatic renal cell carcinoma. Clin Cancer Res. 1998;4:2729-2739.

6. Divgi CR, O'Donoghue JA, Welt S, et al. Phase I clinical trial with fractionated radioimmunotherapy using ${ }^{131} \mathrm{I}$-labeled chimeric G250 in metastatic renal cancer. J Nucl Med. 2004;45:1412-1421. 
7. Divgi CR, Pandit-Taskar N, Jungbluth AA, et al. Preoperative characterisation of clear-cell renal carcinoma using iodine-124-labelled antibody chimeric G250 $\left({ }^{124} \mathrm{I}-\mathrm{cG} 250\right)$ and PET in patients with renal masses: a phase I trial. Lancet Oncol. 2007;8:304-310.

8. Oosterwijk E, Bander NH, Divgi CR, et al. Antibody localization in human renal cell carcinoma: a phase I study of monoclonal antibody G250. J Clin Oncol. 1993;11:738-750.

9. van Dijk J, Oosterwijk E, van Kroonenburgh MJ, et al. Perfusion of tumorbearing kidneys as a model for scintigraphic screening of monoclonal antibodies. J Nucl Med. 1988;29:1078-1082.
10. Steffens MG, Boerman OC, Oosterwijk-Wakka JC, et al. Targeting of renal cell carcinoma with iodine-131-labeled chimeric monoclonal antibody G250. J Clin Oncol. 1997;15:1529-1537.

11. Linden HM, Stekhova SA, Link JM, et al. Quantitative fluoroestradiol positron emission tomography imaging predicts response to endocrine treatment in breast cancer. J Clin Oncol. 2006;24:2793-2799.

12. Mortimer JE, Dehdashti F, Siegel BA, Katzenellenbogen JA, Fracasso P, Welch MJ. Positron emission tomography with 2-[ $\left.{ }^{18} \mathrm{~F}\right]$ fluoro-2-deoxy-D-glucose and $16 \alpha-\left[{ }^{18} \mathrm{~F}\right]$ fluoro-17 $\beta$-estradiol in breast cancer: correlation with estrogen receptor status and response to systemic therapy. Clin Cancer Res. 1996;2:933-939. 